\title{
Microbial Keratitis
}

\author{
Namrata Sharma ${ }^{\otimes}$ \\ All India Institute of Medical Sciences, India
}

Microbial keratitis is an ocular emergency with a potential for blinding complications. Every year, approximately 1.5-2 million cases of corneal blindness are attributed to microbial keratitis. The incidence and etiological factors vary widely, based on the geographical location and the overall economic status of the nations. Preservation of structural anatomy and visual functions requires timely diagnosis and initiation of appropriate antimicrobial therapy. Over the past two decades, the management of microbial keratitis has seen tremendous changes in terms of better understanding and development of various in vitro infection models and treatment therapies such as drug-eluting contact lenses, microneedle patches, and ocular inserts, in an attempt to improve the efficacy and reduce the toxicity of antimicrobial drugs. However, apart from various developments, we have also seen the emergence of multi drug resistant organisms implicated in the causation of bacterial keratitis, largely attributed to their indiscriminate use.
Managing infections caused by the organisms which are resistant to even the newer antibiotics such as imipenem and meropenem, is an important cause for concern.

Fungal keratitis is another form of keratitis, mostly encountered in nations with a high prevalence of agricultural activities. Managing these eyes is a challenge in itself since a lot of patients are started on corticosteroids early on which adversely affects the prognosis and poor corneal penetration of commonly used antifungal medications. Newer formulations and drug delivery systems in the form of liposomes and nanoparticles are underway and hold promise for better treatment of these infections. The use of molecular methods for identification of fungal isolates has helped in the identification of several uncommon aseptate organisms which were once considered to be fungi, but non-responsive to routine antifungal therapy. One such uncommon pathogen belongs to the

\section{Corresponding Author}

Namrata Sharma, MD, DNB, MNAMS

Honorary General Secretary, All India Ophthalmological Society,

Professor, Cornea \& Refractive Surgery Services,

Dr. Rajendra Prasad Centre for Ophthalmic Sciences,

All India Institute of Medical Sciences,

New Delhi, India.

E-mail: namrata.sharma@gmail.com
Access this article online

Website: www.nepjol.info/index.php/NEPJOPH

DOI: https://doi.org/10.3126/nepjoph.v13i2.39755

Copyright $\odot 2021$ Nepal Ophthalmic Society

ISSN: 2072-6805, E-ISSN: 2091-0320

This work is licensed under a Creative Commons

Attribution-NonCommercial-NoDerivatives 4.0

International License (CC BY-NC-ND). 
Pythium genus of Oomycota division. These cases were seen to respond to the oxazolidinone class of antibacterials including Linezolid and the macrolide group of antibiotics.

The acanthamoeba keratitis is another sight threatening infection of the cornea, which is usually encountered in association with contact lens use. Establishing a correct diagnosis remains a real challenge in these cases, the majority being wrongly managed as viral keratitis. The disease runs a long-standing course with slow response to medications and risk of medication induced toxicity.

Microsporidial keratitis, is another emerging corneal infection seen in immunocompromised patients in the form of punctate epithelial keratitis and in immunocompetent patients as a stromal disease. The epithelial disease is self- limiting, the stromal disease however usually does not respond to medical therapy and almost always requires keratoplasty. Establishing correct diagnosis of the stromal variant is a yet another challenge, since patients are wrongly treated on lines of viral keratitis, prior to their presentation altering the initial clinical picture. Overall, successful management of cases with microbial keratitis depends on timely diagnosis and initiation of appropriate therapy by having a strong index of suspicion for a particular form of disease. Delayed referrals and starting antimicrobial and corticosteroid therapy before microbiologic confirmation of the organism are the predominant factors responsible for adverse outcomes. 